\title{
STRUCTURES OF THERMODYNAMIC FUNCTIONS FOR $S$-PAIRED FERMI SYSTEMS IN PARAMETRIC EQUATIONS APPROACH
}

\author{
M. Mulak and R. GonczareK \\ Institute of Physics, Technical University of Wroclaw \\ Wybrzeże Wyspiańskiego 27, 50-370 Wrocław, Poland
}

(Received February 1, 1996)

\begin{abstract}
The general method of investigation of $S$-paired Fermi systems is given and discussed as generalization of parametric equation for the energy gap. The additional parametric equations for thermodynamic potential and heat capacity are obtained as functions of introduced parameters. The detailed calculations are given for a few exemplary chosen magnetic fields and superflows. Moreover, we consider the case of so-called statistical spin liquid, which constitutes non-BCS model.
\end{abstract}

PACS numbers: $74.20 .-z$

\section{Introduction}

The purpose of this paper is an extension of our considerations contained in [1] and formulation of the general methods of investigations of $S$-paired Fermi systems. We extended our results for all these systems, for which the gap equation can be written down in form (1). The class of such systems is quite wide, because it includes all BCS models i.e. enriched by presence of magnetic fields and superflows [2], as well as non-BCS model of statistical spin liquid, formulated by Spalek et al. [3-6] and developed by Czerwonko [7]. We also employed results of Czerwonko [7, 8] to expose the main results of a statistical spin liquid and to illustrate advantage of the established methods.

\section{The general results}

In paper [1] we considered a wide class of $S$-paired quasiparticle Fermi systems (superconductors) where the gap equation can be written in the following general form:

$$
1=\lambda \int_{0}^{\omega_{\mathrm{D}}} \frac{\mathrm{d} \xi}{\sqrt{\xi^{2}+\Delta^{2}(T)}} F\left(\frac{\xi}{2 T}, \frac{\Delta(T)}{2 T}, \frac{a}{2 T}, \frac{b}{2 T}\right),
$$


where $\lambda=\frac{1}{2} \nu(0) g$ is dimensionless coupling parameter, and $a$ and $b$ denote parameters connected with external fields e.g. a magnetic field and a superflow. We also assume that asymptotic properties of the $F\left(l_{1}, l_{2}, l_{3}, l_{4}\right)$ function are analogous to those for BCS case. It tends to a constant value equal to $F_{\infty}$ for large $l_{1}$ or $l_{2}$ and to zero for small $l_{1}$ and $l_{2}$.

We also introduce the same notation as in [1], which allows us to facilitate calculations. The applied dimensionless quantities have the form

$$
U=\frac{\xi}{T_{\mathrm{c}}}, \quad X=\frac{T}{T_{\mathrm{c}}}, \quad Y=\frac{\Delta(T)}{T_{\mathrm{c}}}, \quad A=\frac{a}{T_{\mathrm{c}}}, \quad B=\frac{b}{T_{\mathrm{c}}},
$$

or more convenient

$$
\begin{aligned}
u & =\frac{U}{2 X}=\frac{\xi}{2 T}, & \tau & =\frac{Y}{2 X}=\frac{\Delta(T)}{2 T}, \\
\alpha & =\frac{A}{2 X}=\frac{a}{2 T}, & \beta & =\frac{B}{2 X}=\frac{b}{2 T},
\end{aligned}
$$

which extremely simplify the problem under consideration.

In order to consider the main properties of the system, i.e. the stability, phase transitions and their type, heat capacity and form of energy gap, we employ a standard formula deriving the thermodynamic potential difference between the superconducting state and the normal state, which can be written in the form

$$
\Delta \Omega=\nu(0) T_{\mathrm{c}}^{2} \int_{0}^{Y}\left(\frac{\mathrm{d}}{\mathrm{d} Y^{\prime}} \frac{1}{\lambda}\right)\left(Y^{\prime}\right)^{2} \mathrm{~d} Y^{\prime},
$$

where $\nu(0)$ denotes the density of states on the Fermi surface and $\lambda$ is a functional of the energy gap. In the generalized case under consideration it can be taken in the form (cf. [1])

$$
\begin{aligned}
\frac{1}{\lambda}= & \ln \left(\frac{2 \omega_{\mathrm{D}}}{T_{\mathrm{c}}}\right) F_{\infty}-\ln (Y) F_{\infty} \\
& -\int_{0}^{\infty} \mathrm{d} u\left[\ln \left(u+\sqrt{u^{2}+\tau^{2}}\right)-\ln \tau\right] \frac{\partial}{\partial u}\left[F(u, \tau, \alpha, \beta)-F_{\infty}\right],
\end{aligned}
$$

or equivalently

$$
\begin{aligned}
\frac{1}{\lambda}= & \ln \left(\frac{2 \omega_{\mathrm{D}}}{T_{\mathrm{c}}}\right) F_{\infty}-\ln (2 X) F_{\infty}-\ln (\tau) F(0, \tau, \alpha, \beta) \\
& -\int_{0}^{\infty} \mathrm{d} u \ln \left(u+\sqrt{u^{2}+\tau^{2}}\right) \frac{\partial}{\partial u} F(u, \tau, \alpha, \beta) .
\end{aligned}
$$

Equation (4) after substituting (5) and (6) can be reduced to two entirely equivalent forms

or

$$
\frac{\Delta \Omega}{\nu(0) T_{\mathrm{c}}^{2} F_{\infty}}=-\frac{1}{4} Y^{2}-4 X^{2} \int_{0}^{\tau} \mathrm{d} \tau^{\prime} \tau^{\prime} \ln \frac{Y\left(\tau^{\prime}\right)}{Y(\tau)}
$$

$$
\frac{\Delta \Omega}{\nu(0) T_{\mathrm{c}}^{2} F_{\infty}}=-4 X^{2} \int_{0}^{\tau} \mathrm{d} \tau^{\prime} \tau^{\prime} \ln \frac{X\left(\tau^{\prime}\right)}{X(\tau)}
$$

where now $Y$ and $X$ must be considered as a function of $\tau$. Note that $\tau$ becomes an independent parameter which can vary from zero $\left(T=T_{c}\right)$ to infinity $(T=0)$. 
Applying the well-known relations between entropy, heat capacity and thermodynamic potential [9] i.e.

$$
\Delta S=-\frac{\partial \Delta \Omega}{\partial T}, \quad C_{\mathrm{s}}-C_{\mathrm{n}}=-T \frac{\partial^{2} \Delta \Omega}{\partial T^{2}},
$$

from Eq. (8) we obtain

$$
\begin{aligned}
& S_{\mathrm{s}}-S_{\mathrm{n}}=\Delta S=8 \nu(0) T_{\mathrm{c}} F_{\infty} X(\tau) \int_{0}^{\tau} \mathrm{d} \tau^{\prime} \tau^{\prime}\left[\ln \frac{X\left(\tau^{\prime}\right)}{X(\tau)}-\frac{1}{2}\right] \\
& C_{\mathrm{s}}-C_{\mathrm{n}}=8 \nu(0) T_{\mathrm{c}} F_{\infty} X(\tau)\left\{\int_{0}^{\tau} \mathrm{d} \tau^{\prime} \tau^{\prime}\left[\ln \frac{X\left(\tau^{\prime}\right)}{X(\tau)}-\frac{3}{2}\right]-\frac{1}{2} \frac{X(\tau) \tau}{\partial X(\tau) / \partial \tau}\right\}
\end{aligned}
$$

where for $\alpha=\beta=0$ we have

$$
S_{\mathrm{n}}=C_{\mathrm{n}}=\frac{1}{3} \pi^{2} \nu(0) T .
$$

Note that Eq. (10) can be also rewritten in the form

$$
C_{\mathrm{s}}-C_{\mathrm{n}}=\Delta S-\nu(0) T_{\mathrm{c}} F_{\infty} Y \frac{\partial Y}{\partial X} \text {. }
$$

Equations (7)-(11) together with the gap equations defined in [1] constitute the set of the most general equations, whose solutions allow us to derive the properties of a $S$-paired system connected with stability, phase transitions, critical temperature and low temperature behaviour of the heat capacity.

\section{Application for general BCS case}

Let us employ the developed method for the general BCS case, when the magnetic field and superflow is taken into account.

\subsection{Numerical results}

We exploit the results achieved in [1] and consider the formulas (7) and (8). We substitute the function $Y(T)$ in the form (cf. [1])

$$
\begin{aligned}
& Y(\tau)=\pi \mathrm{e}^{-C} \exp \left[-\frac{1}{2} \int_{0}^{\infty} \mathrm{d} u u \frac{\ln \left(u+\sqrt{u^{2}+\tau^{2}}\right)-\ln \tau}{\sqrt{u^{2}+\tau^{2}}}\right. \\
& \left.\times \sum_{i=0}^{1} \frac{\tanh \left(\sqrt{u^{2}+\tau^{2}}+\omega+(-1)^{i} \chi\right)-\tanh \left(\sqrt{u^{2}+\tau^{2}}-\omega+(-1)^{i} \chi\right)}{2 \omega}\right]
\end{aligned}
$$

where

$$
X(\tau)=\frac{1}{2 \tau} Y(\tau)
$$

and

$$
R(\tau)=\frac{\sqrt{1+4 \tau^{2}}}{2 \tau} Y(\tau)
$$

is a slowly varying function of its arguments. The quantities $\chi$ and $\omega$ represent additional fields and are functions of $\tau$ as given by

$$
\chi=\frac{1}{2} \pi \mathrm{e}^{-C} \frac{\sqrt{1+4 \tau^{2}}}{R(\tau)} h,
$$




$$
\omega=\frac{1}{2} \pi \mathrm{e}^{-C} \frac{\sqrt{1+4 \tau^{2}}}{R(\tau)} v,
$$

where $h=\mu_{\mathrm{B}} H / \Delta(0)$ and $v=p_{\mathrm{F}} V_{\mathrm{s}} / \Delta(0)$ stand for reduced values of the magnetic field and superflow, respectively. Since $R(\tau)$ is a slowly varying function of $\tau$, in order to derive the shape of the energy gap for the fixed magnetic field and superflow we apply self-consistent iteration methods.

In order to evaluate the thermodynamic potential of a superconducting phase we have to reduce its normal contribution according to Eq. (11). The heat capacity can be easily obtained from the formula (cf. [9])

$$
C=-T \frac{\partial^{2} \Omega}{\partial T^{2}}
$$

which in a case of BCS states after substituting (8) transforms to the form

$$
\frac{1}{\nu(0)} C_{\mathrm{s}}=\frac{1}{3} \pi^{2} X(\tau)+8 X(\tau) \int_{0}^{\tau} \mathrm{d} \tau^{\prime} \tau^{\prime} \ln \frac{Y\left(\tau^{\prime}\right)}{Y(\tau)}-4 X(\tau) \tau^{2}-4 \frac{X^{2}(\tau) \tau}{\partial X(\tau) / \partial \tau} .
$$

In Figs. 1-3 the plots of difference of thermodynamic potentials of superconducting and normal states, and the heat capacity of the general BCS state vs. reduced temperature are shown for a few chosen values of $h$ and $v$.
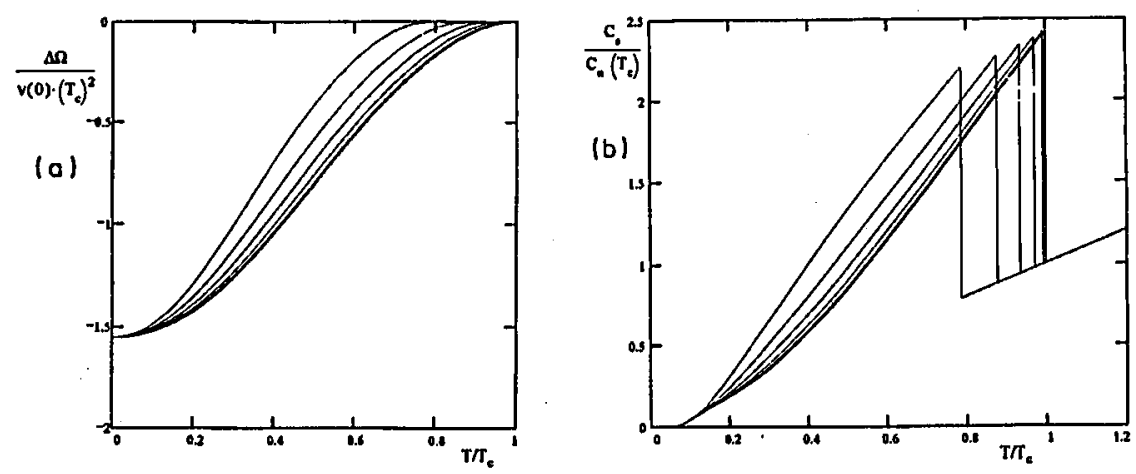

Fig. 1. The exemplary plots of (a) difference of the reduced thermodynamic potential of superconducting general BCS state and the normal state, and (b) heat capacity of the general BCS state vs. the reduced temperature for chosen values of $h$ and $v$. The succeeding curves from down to up correspond to $v=0$ and $h=0,0.1,0.2,0.3,0.4,0.5$, respectively. Note that $\Delta \Omega$ takes its minimum value -1.55 for $T=0$ and the values of $\Delta \Omega$ shift up when the value of $h$ rises. Similarly, the maximum of heat capacity temperature dependence shifts left and takes the following values $\left[h, T / T_{c}, C_{s} / C_{\mathbf{n}}\left(T_{c}\right)\right]:(0,1,2.43)$, $(0.1,0.99,2.42),(0.2,0.97,2.38),(0.3,0.94,2.33),(0.4,0.8,2.26),(0.5,0.79,2.19)$. 

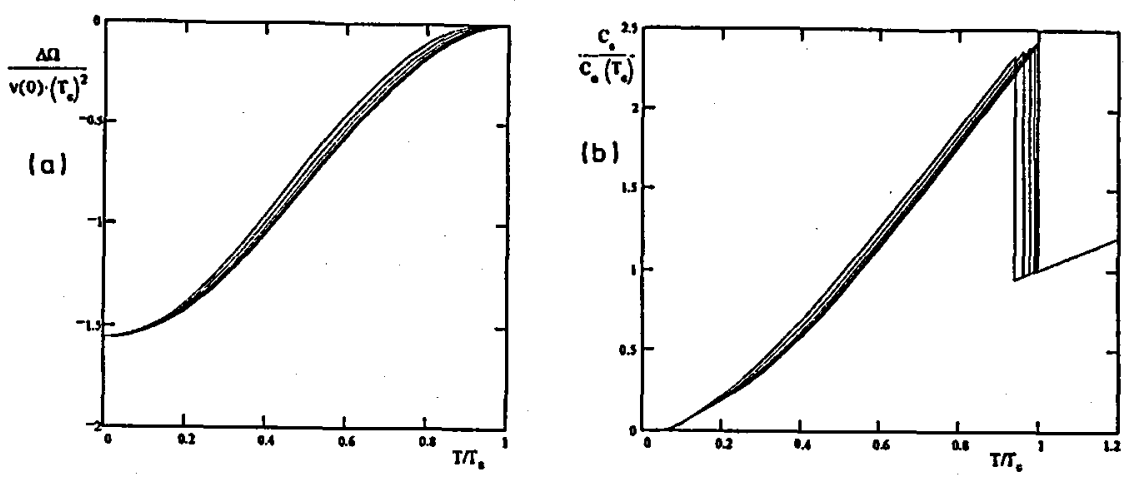

Fig. 2. See description of Fig. 1. Here, the succeeding curves from down to up correspond to $h=0$ and $v=0,0.1,0.2,0.3,0.4,0.5$, respectively. Note that as in Fig. 1 , $\Delta \Omega$ takes its minimum value -1.55 for $T=0$ and the values of $\Delta \Omega$ shift up when the value of $v$ increases, as well as the maximum of heat capacity dependence shifts slightly left and takes the following values $\left[v, T / T_{\mathrm{c}}, C_{\mathrm{s}} / C_{\mathrm{n}}\left(T_{\mathrm{c}}\right)\right]:(0,1,2.43),(0.1,1,2.42)$, $(0.2,0.99,2.42),(0.3,0.98,2.40),(0.4,0.96,2.37),(0.5,0.94,2.34)$. Apparently, the influence of $v$ (for $h=0$ ) is much smaller than the influence of $h($ for $v=0)$.
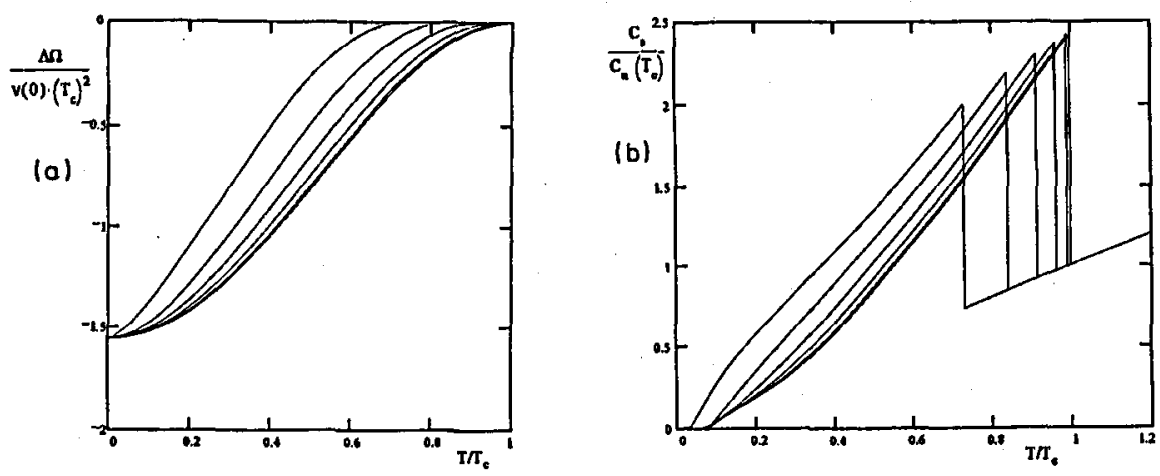

Fig. 3. See description of Fig. 1. In this case the influence both of $v$ and $h$ is taken into account. The succeeding curves from down to up correspond to the following values of $(h, v):(0,0),(0.1,0.1),(0.2,0.2),(0.3,0.3),(0.4,0.4),(0.5,0.5)$, respectively. Note that as in Fig. 1 and Fig. $2, \Delta \Omega$ takes its minimum value -1.55 for $T=0$, and, as above, the value of $\Delta \Omega$ must shift up when the values of $v$ and $h$ increase simultaneously. Similarly the maximum of heat capacity dependence shifts left taking the following values $\left[v, h, T / T_{\mathrm{c}}, C_{\mathrm{s}} / C_{\mathrm{n}}\left(T_{\mathrm{c}}\right)\right]:(0,0,1,2.43),(0.1,0.1,0.99,2.42)$, $(0.2,0.2,0.96,2.37),(0.3,0.3,0.92,2.30),(0.4,0.4,0.84,2.18),(0.5,0.5,0.73,1.99)$. In the latter case $(v=h=0.5)$ the exponential character of the $C_{s}$ plot close to $T=0$ is broken down to the plateau $\Delta \Omega=0$, which is apparently shorter than that in Fig. 1 and Fig. 2. 


\section{Analytical results}

Let us complete the obtained results and consider consistently Eqs. (7) and (8) in the two opposite limits i.e. $\tau=\infty$ and $\tau=0$. In the limit low temperatures when $\tau \rightarrow \infty$ we should apply Eq. (7) and the following formula:

$$
\ln \frac{\Delta(\tau)}{\Delta(0)}=\frac{1}{F_{\infty}} \int_{0}^{\infty} \mathrm{d} u \frac{F(u, \tau, \alpha, \beta)-F_{\infty}}{\sqrt{u^{2}+\tau^{2}}},
$$

which allows us to express $Y$ as a function of $\tau$, then after some calculations Eq. (7) reduces to the form

$$
\frac{\Delta \Omega}{\nu(0) T_{\mathrm{c}}^{2} F_{\infty}}=-\frac{1}{4} Y^{2}(T=0)+8 X^{2} \int_{0}^{\tau} \mathrm{d} \tau^{\prime} \tau^{\prime} \ln \frac{\Delta\left(\tau^{\prime}\right)}{\Delta(0)}
$$

and hence we get

$$
\Omega_{\mathrm{s}}=-\frac{\nu(0)}{4} \Delta^{2}(T=0)-\nu(0) \mathrm{e}^{-\Delta / T} \sqrt{2 \pi \Delta T^{3}} \operatorname{ch} \frac{\mu_{\mathrm{B}} H}{T} \frac{\operatorname{sh}\left(p_{\mathrm{F}} V_{\mathrm{s}} / T\right)}{p_{\mathrm{F}} V_{\mathrm{s}} / T},
$$

then

$$
\Omega_{\mathrm{s}}=-\nu(0)\left\{\frac{\Delta^{2}(T=0)}{4}+[\Delta(T=0)-\Delta] T\right\}
$$

and heat capacity can be approximated by

$$
\begin{aligned}
C_{\mathrm{s}}= & \nu(0) \sqrt{2 \pi \Delta / T^{3}} \mathrm{e}^{-\Delta / T}\left[\left(\Delta^{2}+\mu_{\mathrm{B}}^{2} H^{2}+p_{\mathrm{F}}^{2} V_{\mathrm{s}}^{2}\right) \operatorname{ch} \frac{\mu_{\mathrm{B}} H}{T} \frac{\operatorname{sh}\left(p_{\mathrm{F}} V_{\mathrm{s}} / T\right)}{p_{\mathrm{F}} V_{\mathrm{s}} / T}\right. \\
& +\frac{2 T}{V}\left(\mu_{\mathrm{B}} H p_{\mathrm{F}} V_{\mathrm{s}} \operatorname{sh} \frac{\mu_{\mathrm{B}} H}{T} \operatorname{ch} \frac{p_{\mathrm{F}} V_{\mathrm{s}}}{T}-\mu_{\mathrm{B}} H \Delta \operatorname{sh} \frac{\mu_{\mathrm{B}} H}{T} \operatorname{sh} \frac{p_{\mathrm{F}} V_{\mathrm{s}}}{T}\right. \\
& \left.\left.-p_{\mathrm{F}} V_{\mathrm{s}} \Delta \operatorname{ch} \frac{\mu_{\mathrm{B}} H}{T} \operatorname{ch} \frac{p_{\mathrm{F}} V_{\mathrm{s}}}{T}\right)\right],
\end{aligned}
$$

which in case $H>0$ and $V_{\mathrm{s}}>0$ can be replaced by

$$
C_{\mathrm{s}}=\frac{1}{4} \nu(0) \sqrt{2 \pi \Delta / T p_{\mathrm{F}} V_{\mathrm{s}}}\left(\Delta-\mu_{\mathrm{B}} H-p_{\mathrm{F}} V_{\mathrm{s}}\right)^{2} \mathrm{e}^{-\left(\Delta-\mu_{\mathrm{B}} H-p_{\mathrm{F}} V_{\mathrm{s}}\right) / T} .
$$

In the opposite temperature limit, when $T$ is close to $T_{\mathfrak{c}}$, i.e. $\tau \rightarrow 0$, we get the following relations:

$$
\begin{gathered}
\frac{\Delta \Omega}{T_{\mathrm{c}}^{2} \nu(0)}=-\frac{1}{4 P}\left(1-\frac{T}{T_{\mathrm{c}}}\right)^{2}+\frac{1}{4 P}\left(1-\frac{2}{3} \frac{Q}{P^{2}}\right)\left(1-\frac{T}{T_{\mathrm{c}}}\right)^{3} \\
+M\left(1-\frac{T}{T_{\mathrm{c}}}\right)+2 M\left(1-2 \frac{Q}{P^{2}}\right)\left(1-\frac{T}{T_{\mathrm{c}}}\right)^{2}
\end{gathered}
$$

where (cf. [2])

and the factor

$$
P=\frac{7 \zeta(3)}{8 \pi^{2}}, \quad Q=\frac{93 \zeta(5)}{128 \pi^{4}}
$$

$$
M=\frac{1}{T_{\mathrm{c}}^{2}}\left[\mu_{\mathrm{B}}^{2} H^{2}+\frac{1}{3} p_{\mathrm{F}}^{2} V_{\mathrm{s}}^{2}\right]
$$

expresses the influence of the fields $H$ and $V_{\mathrm{s}}$. 
Differentiating the above formula and after some transformations we find reduced heat capacity of superconductors in a vicinity of $T_{\mathrm{c}}$ in the form

$$
\frac{C_{\mathbf{s}}(T)}{C_{\mathrm{n}}\left(T_{c}\right)}=C_{1}+C_{2} M+C_{3}\left(\frac{T}{T_{\mathrm{c}}}-1\right)
$$

where

$$
\begin{aligned}
& C_{1}=\frac{3}{2 P \pi^{2}}+1=2.426 \\
& C_{2}=\frac{3}{\pi^{2}}\left(6 \frac{Q}{P^{2}}-1\right)=0.938 \\
& C_{3}=\frac{6}{P \pi^{2}}-3 \frac{Q}{P^{3} \pi^{2}}+1=4.762 .
\end{aligned}
$$

\section{Non-BCS model - statistical spin liquid}

The very interesting problem constitutes the non-BCS model formulated by Spałek and Wöjcik [3, 4], and intensively extended by Czerwonko [7, 8], so-called statistical spin liquid.

In this model fermion quasiparticles cannot be situated in all quasiparticles states according to Fermi-Dirac distribution rules. The form of the Hamiltonian introduced in $[3,4]$ decreases the total number of quasiparticles in the same quantum state with arbitrary spins, whereas in the approach developed by Czerwonko [7] their number equals to one. It means that in the space of states the double spin occupancy of the same quasimomentum is forbidden. Such distribution of quasiparticles causes that the creation of the Cooper pairs become possible only if in states with opposite momentum $p$ quasiparticles exist with opposite spins.

Therefore, in this model, the Cooper pairs can appear with some probability, which can be easy derived by means of distribution function. Let us consider now the most important functions which allow us to evaluate properties of a superconducting state. We employ the developed formalism and results obtained by Czerwonko in $[7,8]$. According to [7], the function $F$ can be taken in the form

$$
F[\xi, \Delta(T)]=\sum_{i=0}^{1} \frac{\sinh \left[\frac{1}{T} \sqrt{\xi^{2}+2 \Delta^{2}(T)}\right]}{2 \cosh \left[\frac{1}{T} \sqrt{\xi^{2}+2 \Delta^{2}(T)}\right]+4+3 \exp \left[(-1)^{i} \xi / T\right]}
$$

and now $F_{\infty}=\frac{5}{8}$. Applying the formalism established in [1] and extended in this paper and using the numerical methods, we can easily derive and analyse some characteristic properties of the statistical spin liquid under discussion.

In Fig. 4 we presented the forms of the energy gap, thermodynamic potential difference between $S$-paired and normal states and heat capacity in reduced units as functions of reduced temperature, which have been derived by means of Eqs. (1), (8), (10), respectively.

The obtained curves reveal amazing properties of the statistical spin liquid model. According to obtained numerical results the system shows anomalous properties in the region of temperatures from 0 to 0.53 (in reduced scale), since 

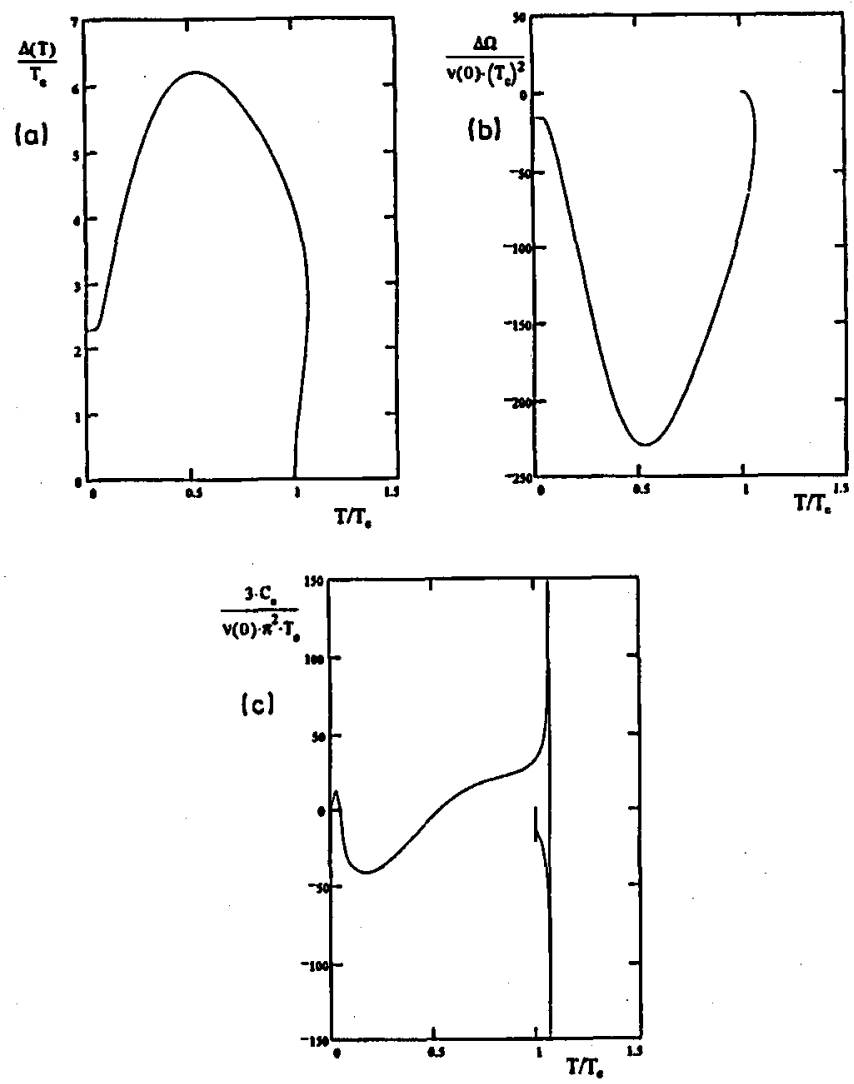

Fig. 4. (a) Energy gap for the statistical spin liquid model vs. reduced temperature. $\Delta_{\max } / T_{\mathrm{c}}=6.20$ for $T / T_{\mathrm{c}}=0.53$. Other charactcristic points of the plot are situated as follows: $\Delta(T=0)=2.29, \Delta\left(T / T_{\mathrm{c}}=1\right)=4.17, \Delta\left(T / T_{\mathrm{c}}=\right.$ $1.067)=2.73$, and $\Delta$ becomes increasing for $T / T_{c}=0.045$. (b) The reduced thermodynamic potential difference for the statistical spin liquid model vs. the reduced temperature. Minimum of $\Delta \Omega$ achieved for $T / T_{\mathrm{c}}=0.53$ is equal to -230. Other characteristic points are situated as follows: $\Delta \Omega(T=0)=-15.6$ (and $\Delta \Omega<-15.6$ starting from 0.045), $\Delta \Omega\left(T / T_{c}=1\right)=-83.6$ and 0 (double-value function), $\Delta \Omega\left(T / T_{c}=1.067\right)=-26.5, \Delta \Omega\left(T / T_{\mathrm{c}}=1.016\right)=0$, $\Delta \Omega\left(T / T_{\mathrm{c}}=1.006\right)=0.39$. (c) The heat capacity plot for the statistical spin liquid model vs. the reduced temperature. For the characteristic points it achieves the following values: $C_{\mathrm{s}}\left(T / T_{\mathrm{c}}=0.53\right)=0, C_{\mathrm{s}}(T=0)=0$ and $C_{\mathrm{s}}\left(T / T_{\mathrm{c}}=0.049\right)=0$, $C_{s}\left(T / T_{\mathrm{c}}=0.029\right)=12.9$ (local maximum), $C_{\mathrm{s}}\left(T / T_{\mathrm{c}}=0.17\right)=-44.4$ (local minimum), $C_{\mathrm{s}}\left(T / T_{\mathrm{c}}=1\right)=33.5$ and $C_{\mathrm{s}}\left(T / T_{\mathrm{c}}=1.067\right)$ tends to infinity. Negative values of $C_{\mathrm{s}}$ seem to be specific and unusual, at the first view, feature of the considered theory (see Sec. 5 ).

energy gap and thermodynamical potential are increasing and decreasing functions of temperature, respectively. Nevertheless, it could be connected with additional creation of Cooper pairs. 
Moreover, in temperature region from 0.049 to 0.53 the heat capacity becomes negative, which is non-physical, and the system is quite unstable. This time it can be interpreted as follows: since the number of Cooper pairs increases together with temperature, the thermodynamic potential decreases and the system emits an excessive energy, i.e. heat, which additionally warms the system. Hence, negative values of the heat capacity appear.

In the region temperatures above 0.53 system shows reasonable behavior. However the paired state can exist also for temperatures over 1 until 1.067. For higher temperatures thermodynamic potential difference equals zero, so the energy gap must drop to zero. This property determines the type of phase transition to the normal phase, which must be type I. It also explains singularity of the heat capacity at phase transition point. Let us notice that anomalous behaviour of the heat capacity appears also for $T / T_{c}=1$, which becomes double-value function of temperature.

Concluding we emphasize that though we presented the detailed interpretation of the obtained results, the statistical spin liquid model proposed by Spatek et al. $[3,1]$ seems to be non-physical, which never can be realized in real systems. Our arguments support this objection. Moreover, the established formalism allows us to derive the properties of the $S$-paired systems, and can be exploited in order to verify other models.

\section{Conclusions}

The results presented in this paper and paper [1] constitute a convenient set of formulas allowing to investigate any $S$-paired system of fermions, for which the gap equation can be written in form (1). The performed calculations allowed us to make essential progress in research of the generalized BCS systems, when the presence of a magnetic field and superflow is taken into account. The obtained results (Figs. 1-3) reveal the sensibility of BCS systems on perturbation given rise by the magnetic field or superflow.

The developed method allows us also to derive and examine the properties of the statistical spin liquid in approach established by Czerwonko [7].

We emphasize that the obtained results concern only one exceptionally favored model of the statistical spin liquid for which the doubly occupied states are strictly forbidden as a result of infinite energetic discrimination.

The accurate investigations show qualitative differences in comparison with BCS systems. Moreover, it was recently shown that the statistical spin liquid constitutes an universality class of the one-dimensional IIubbard model with the infinitely strong Coulomb repulsion [10,11]. Therefore, there exists a wide class of models, which can be investigated using developed method.

This research has been supported in part by grant 2 P 30212506 of the Committee for Scientific Research.

\section{References}

[1] R. Gonczarek, M. Mulak, Acta Phys. Pol. A 86, 1007 (1994).

[2] R. Gonczarek, Acta Phys. Pol. A 71, 59 (1987). 
[3] J. Spalek, W. Wójcik, Phys. Rev. B 37, 1532 (1988).

[4] J. Spalek, Phys. Rev. B 40, 5180 (1989).

[5] K. Byczuk, J. Spatek, Phys. Rev. B 50, 11403 (1994).

[6] K. Byczuk, J. Spatek, Acta Phys. Pol. A 85, 337 (1994).

[7] J. Czerwonko, Physica $C$ 235-240, 2307 (1994).

[8] J. Czerwonko, Mol. Phys. Rep., in press.

[9] A.A. Abrikosov, Fundamental Theory of Metals, North-Holland, Amsterdam 1988. [10] J. Spalek, Acta Phys. Pol. A 85, 39 (1994).

[11] J. Spalek, W. Wójcik, Acta Phys. Pol. A 85, 357 (1994). 